\title{
Método de los promedios anuales en el monitoreo de los cambios de cobertura por deforestación usando el sensor MODIS
}

\author{
The average annual changes deforestation monitoring method in coverage using the MODIS \\ model
}

\author{
Yonatan Tarazona Coronel
}

\begin{abstract}
Laboratorio de Teledetección, Facultad de Ciencias Físicas, Universidad Nacional Mayor de San Marcos, Ciudad Universitaria, Lima-Perú
\end{abstract}

DOI: https://doi.org/10.33017/RevECIPeru2014.0007/

\section{Resumen}

La deforestación de los bosques, a través de la expansión agrícola, la conversión de áreas boscosas a pasturas, el desarrollo de infraestructura, la tala destructiva, los incendios, etc. representan casi el $20 \%$ de las emisiones de gases de efecto invernadero a nivel mundial, más que todo el sector de transporte global y sólo superada por el sector energético. Por lo tanto, con el fin de limitar los impactos del cambio climático dentro de los límites, que la sociedad razonablemente es capaz de tolerar, las temperaturas medias globales deben estabilizarse dentro de los dos grados centígrados. Este objetivo será prácticamente imposible de lograr sin reducir las emisiones del sector forestal, además de otras acciones de mitigación (REDD+).

Si bien es cierto existen metodologías para el monitoreo de la deforestación, la mayoría de estos métodos, por no decir todos, requieren la designación del usuario de una definición de umbral que permita clasificar e identificar un cambio en el uso del suelo por deforestación. La determinación de los umbrales añade un coste significativo a los esfuerzos crecientes de detección de cambios en las distintas regiones y limita el estudio en diversas regiones que sufren cambios en la cobertura y que es necesario cuantificarlo. Se necesita, entonces, un análisis histórico a partir de datos de satélite archivado para modelar el comportamiento normal y el comportamiento anormal, es decir, la perturbación (Hargrove et al. 2009). Hay una necesidad crítica que permite el análisis de series de tiempo independiente de umbrales o definiciones para detectar alteraciones específicas. Este trabajo presenta un método que realiza, en un primer paso, un preprocesamiento de series de tiempo de índices EVI-MODIS antes de detectar y cuantificar los cambios de cobertura por deforestación. La metodología básicamente consiste en tener la serie de tiempo completa luego de interpolar los datos faltantes al filtrar los pixeles perturbados con la banda de fiabilidad del producto MOD13Q1. Luego de tener la serie completa para cualquier pixel, se promedia cada año los datos de tal manera que tengamos 13 valores desde el 2001-2013. Finalmente utilizaremos los límites de control de la estadística clásica para observar qué promedio está fuera de control y detectarlo como un cambio de uso por deforestación.

Descriptores. Perturbación, Deforestación, EVI, Annual Averages.

\section{Abstract}

The clearing of forests, through agricultural expansion, conversion of forests to pasture, infrastructure development, destructive logging, fires, etc. represent nearly $20 \%$ of emissions of greenhouse gases worldwide, more than the entire global transportation sector and second only to the energy sector. Therefore, in order to limit the impacts of climate change within limits that society can tolerate reasonably, global average temperatures 
must be stabilized within two degrees Celsius. This objective will be virtually impossible to achieve without reducing emissions from the forest sector as well as other mitigation (REDD +).

While there are methodologies for monitoring deforestation, most of these methods, if not all, require the user designation of a definition of threshold for classifying and identifying a change in land use from deforestation. Determining thresholds adds significantly to the increased efforts to detect changes in the regions cost and limits the study in various regions that undergo changes in coverage and the need to quantify it. Is needed, then, a historical analysis from archived satellite data to model the normal behavior and abnormal behavior, ie the disturbance (Hargrove et al. 2009). There is a critical need to allow independent analysis of time series of thresholds or definitions to detect specific abnormalities.

This paper presents a method that takes as a first step, a pre-processing time series of MODIS-EVI indexes before detecting and quantifying changes in coverage due to deforestation. The methodology basically involves having the entire time series then interpolate the missing data by filtering pixels disturbed with the band MOD13Q1 product reliability. After having the complete set for every pixel, every year averaged data so we have 13 values from 2001 to 2013 . Finally use the control limits of classical statistics to see what the average is out of control and detect a change of use from deforestation.

\section{Keywords. Disturbance, Deforestation, EVI, Box-Jenkins}

\section{Introducción}

Los bosques tropicales proporcionan diversos servicios a los ecosistemas, tanto a escala global y local (Kremen y Ostfeld 2005). Contienen más especies que cualquier otro ecosistema en tierras emergidas (Gibson et al. 2011) y son grandes sumideros de carbono (Pan et al. 2011). A nivel local, los bosques tropicales tienen la capacidad de regular el suministro de agua y proporcionar agua de alta calidad a las poblaciones de los alrededores (Bradshaw et al. 2007). Por lo tanto, la deforestación tropical es responsable no sólo de una importante reducción de la biodiversidad (Gibson et al 2011.), sino también una proporción considerable (6-17\%) de las emisiones globales de dióxido de carbono que afectan el cambio climático (IPCC 2007; Baccini et al. 2012) y es el primer paso hacia la desertificación de la tierra (Geist 2005; Xu et al 2011).

La región de San Martín es una de las regiones más deforestadas del Perú. Su tasa de deforestación es la más alta con 35, 278.85 ha/año (MINAM 2012). Siendo las causas principales: la quema de bosques con fines agropecuarios, la producción de carbón, leña y la explotación de madera con fines comerciales.

Es una región propicia para determinar la precisión de esta nueva metodología que detecta cambios grandes a mediano y largo plazo y no cambios pequeños en el corto plazo, siendo este el corazón de la metodología.

\section{Marco Teórico}

\subsection{Serie de Tiempo}

Se llama serie de tiempo a un conjunto de observaciones sobre valores que toma una variable cuantitativa en diferentes momentos de tiempo a iguales intervalos.

\section{¿Qué se analiza en una serie de Tiempo?}

- Componentes de una serie de Tiempo

- Tendencia

- Ciclo

- Estacionalidad

- Movimiento aleatorio (ruido blanco)

a) Tendencia. Patrón de evolución sostenido a mediano y largo plazo de la serie Crecer, decrecer o estable.

b) Ciclo. Movimiento oscilatorio por encima y por debajo de la tendencia de una serie temporal.

c) Estacionalidad. Oscilaciones de una serie temporal que se completa dentro de un

d) Aleatorio. Oscilación de una serie temporal que se atribuyen a factores fortuitos, aleatorios y esporádicos. 


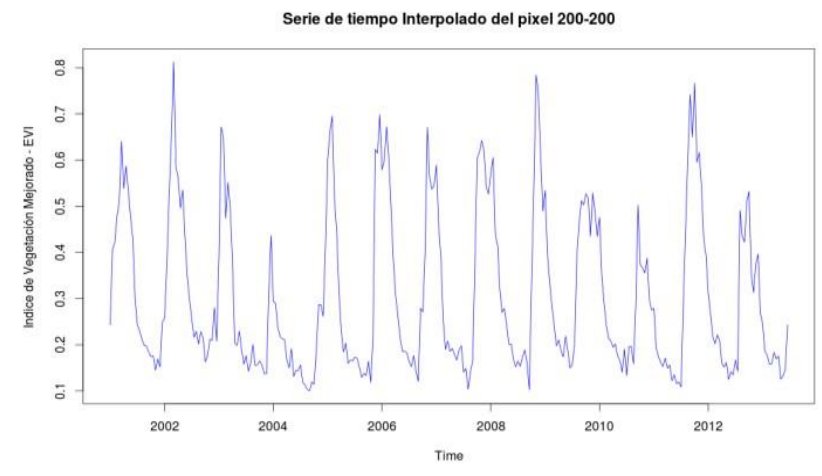

Figura 1: Serie de Tiempo del Índice de Vegetación Mejorado del sensor MODIS.

\subsection{Gráficos de Control}

En un gráfico de control se representa gráficamente una característica calculada a partir de muestras del producto, en función de las diferentes muestras. La gráfica tiene una línea central que simboliza el valor medio de la característica. Finalmente, otras dos líneas (los límites superior e inferior de control) flanquean a la anterior a una distancia determinada. Estos límites son escogidos de manera que si el proceso está bajo control, casi la totalidad de los puntos muestrales se hallen entre ellos. Así, un punto que se encuentra fuera de los límites de control se interpreta como una evidencia de que el proceso está fuera de control.

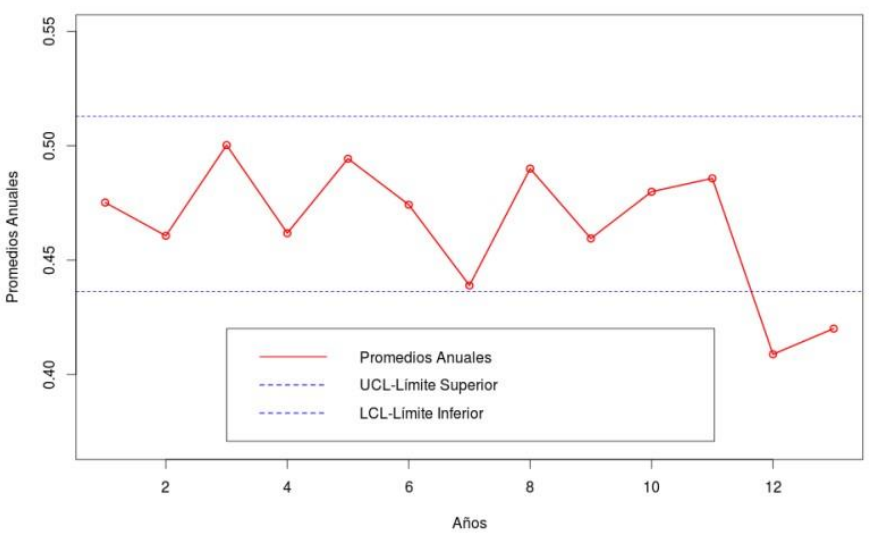

Figura 2: Gráfico de control en donde se evidencia que los promedios de los años 2012 y 2013 están fuera del límite inferior, lo que indica un cambio de uso del suelo.

Un modelo general de gráfico de control se basa en conceptos y resultados estadísticos como sigue:
Donde

$$
\mathrm{LCL}=\mu-k \sigma,
$$

$\mu$ : La media del proceso $\sigma: \mathrm{La}$ desviación estándar del proceso

y $k$ es la distancia del valor central a los limites superior e inferior. Habitualmente o comúnmente utilizan $k=3$, pero esto depende del comportamiento de los datos que tengamos por lo que podemos utilizar $k=5,6$, etc.

\section{Metodología}

En primera instancia se tuvo que descargar los tiles h09v09 y h10v09 del índice EVI del producto MOD13Q1 del sensor MODIS para luego apilarlos uno encima de otro en el orden cronológico (fig.3). Una vez apiladas las imágenes se debe filtrar aquellos pixeles malos que pueden ser nubes, glaciares (si es que lo hubiera), etc. Para esto es necesario utilizar la banda de fiabilidad que viene al descargar los tiles del producto.

En la versión 5 de las imágenes MODIS se incorpora una capa (pixel reliability) que contiene información resumida sobre la fiabilidad de los datos, en dónde:

$0=$ Fiable ( $\sin$ nubes, sin nieve, sin carga atmosférica de aerosoles)

$1=$ Semi-fiable (sin nubes, sin nieve, el objetivo es visible y las correcciones atmosféricas han mejorado la calidad del dato)

$2=$ Nieve (superficie cubierta total 0 parcialmente por nieve)

$3=$ Nubes (presencia total o parcial de nubes) 4 = Sin datos (información no procesada por razones diversas).

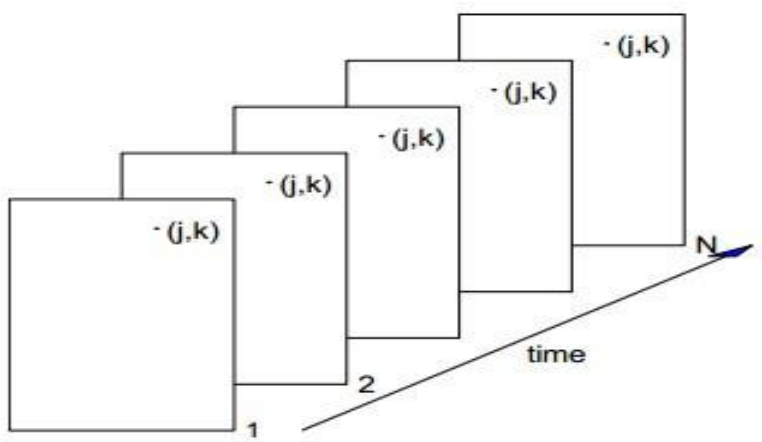

$$
\begin{aligned}
& \mathrm{UCL}=\mu+k \sigma, \\
& \mathrm{LC}=\mu,
\end{aligned}
$$


Figura 3: Imágenes ordenadas cronológicamente donde $N=2013$.

Es necesario que se use esta información para eliminar datos que pudieran estar afectados por la cobertura de nieve o la presencia de nubes antes de proceder a su análisis (Luis Cayuela-2010).

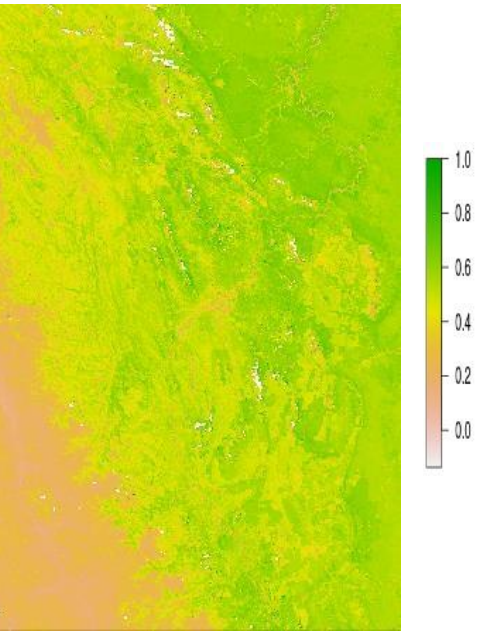

(a)

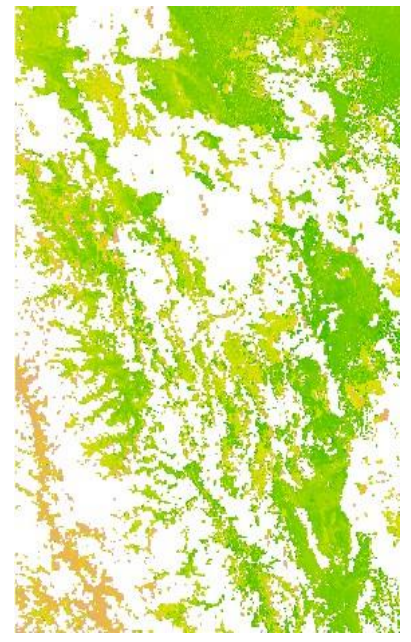

(b)
Figura 4: En la figura (a) se muestra una imagen original sin el filtrado de píxeles malos y en la figura (b) se muestra la imagen luego de filtrar los pixeles malos mediante la banda de fiabilidad.

Una vez eliminado los píxeles malos, aquellas series de tiempo de píxeles que tengan datos "NA", se debe interpolar de tal manera que tengamos la serie completa para aplicar la metodología de Promedios Anuales. Como las imágenes están cada 16 días de resolución temporal-esto nos da 23 imágenes al año; por lo que se tendrá promediar estas 23 de tal manera que tendremos 13 promedios muestrales para el análisis.

Se muestra el procedimiento de los límites de control para un pixel deforestado el año 2013. Este procedimiento se realiza tantas veces la cantidad de pixel que tenga una imagen satelital, lógicamente es necesario la programación para analizar pixel a pixel.

Para el pixel $\mathrm{i}=3, \mathrm{j}=70$

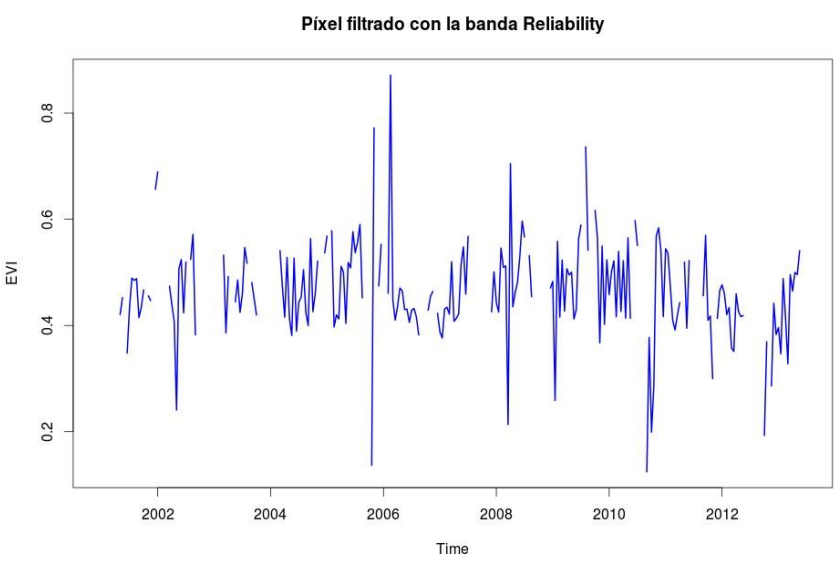

Figura 5: Luego de filtrar los valores mediante la banda de fiabilidad - Pixel Reliability

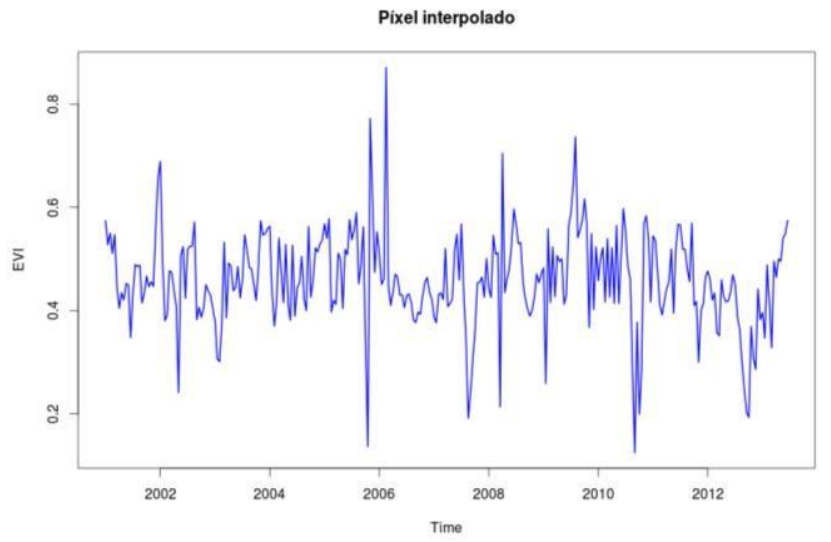

Figura 6: Luego de interpolar los valores faltantes para completar la serie.

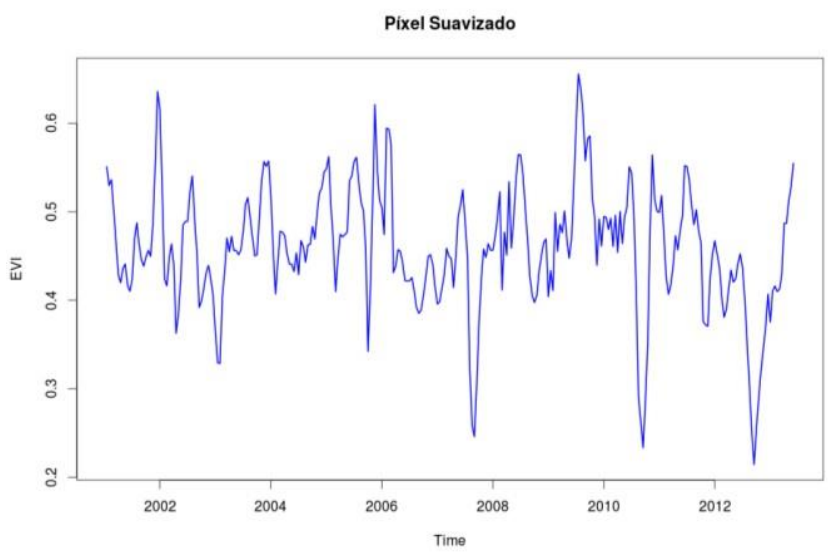

Figura 7: Luego suavizar la serie para minimizar algún valor anómalo en los datos.

Para la posición $\mathrm{i}=3, \mathrm{j}=70$ tenemos 299 valores distribuidos 23 cada año.

Se procede a promediar estos valores anuales de tal manera que tengamos 13 datos desde el 20012013 como se muestra a continuación. 
Promedios $=\mathrm{p} 1, \mathrm{p} 2, \mathrm{p} 3, \ldots ., \mathrm{p} 12, \mathrm{p} 13$.

$\mu$ : $\quad$ La media de estos promedios anuales a excepción del último año a analizar. $\sigma$ : La desviación estándar de estos promedios $\mathrm{n}$ : la cantidad de datos

Para la posición $\mathrm{i}=1, \mathrm{j}=70$ tenemos lo siguiente:

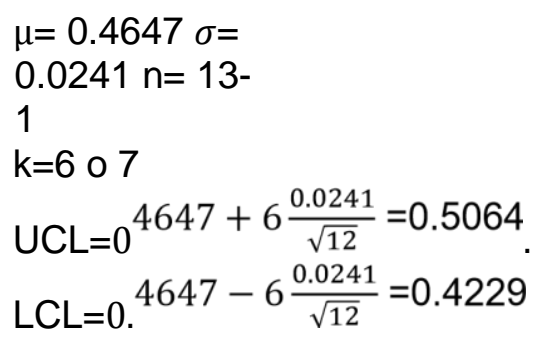

La grafica se muestra a continuación.

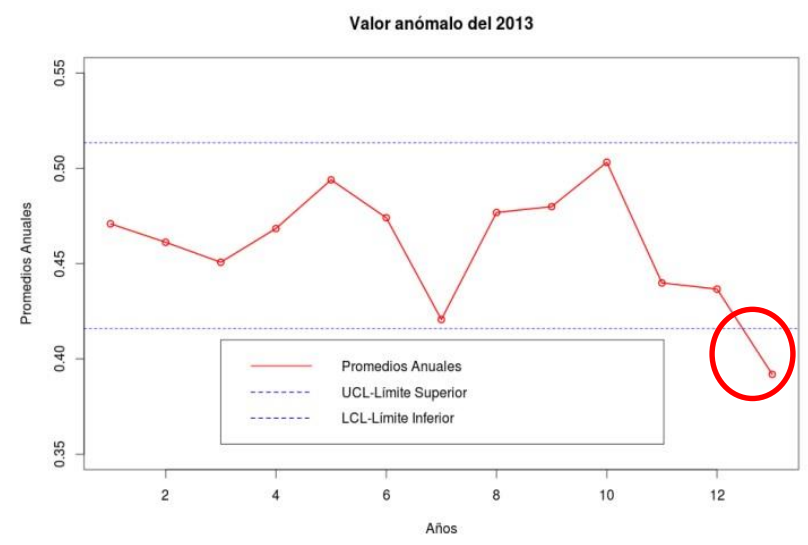

Figura 8: Este valor fuera de control es detectado como deforestación, ya que los valores de verdor están descendiendo lo que indica que hay un cambio de uso del suelo por otra actividad.

\section{Resultados}

Se ha probado el método para la provincia de Moyobamba en la Región de San Martín para el año 2012 y 2013, para esto se ha utilizado imágenes Landsat TM y Landsat 8 de los años 2010 y 2013 de tal manera que con una imagen de $30 \mathrm{~m}$ de resolución espacial podamos apreciar mejor los resultados.

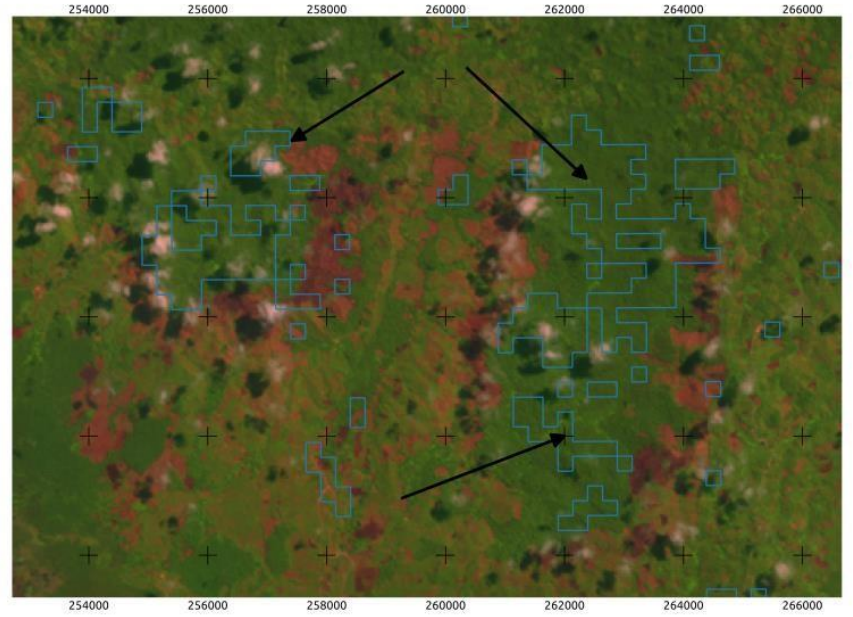

Figura 9: Esta es una imagen del año 2010 en donde se muestra que las áreas dentro de los polígonos de color azul están intactas.

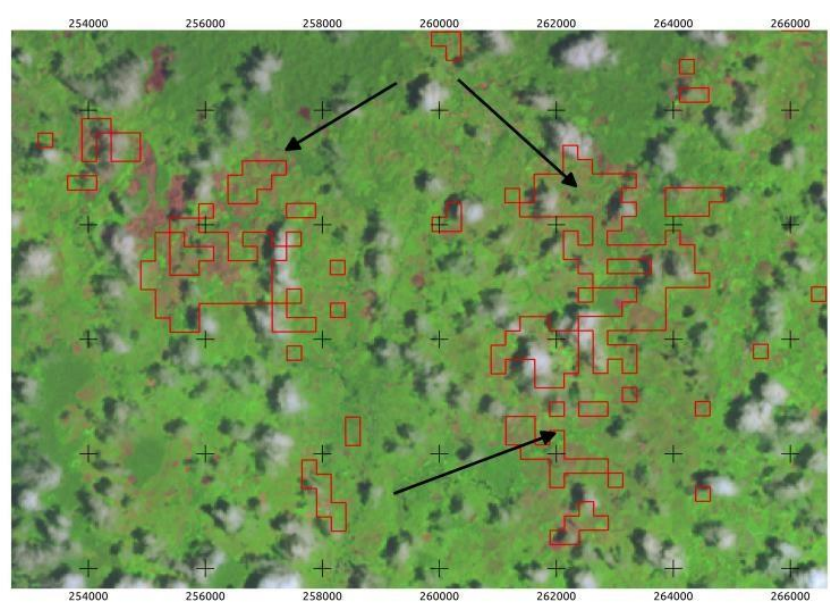

Figura 10: Esta es una imagen del año 2013 en donde se muestra que las áreas dentro de los polígonos de color azul ya están deforestadas y fueron detectadas por Los Promedios Anuales.

Veamos los cambios en otra posición del área de estudio.

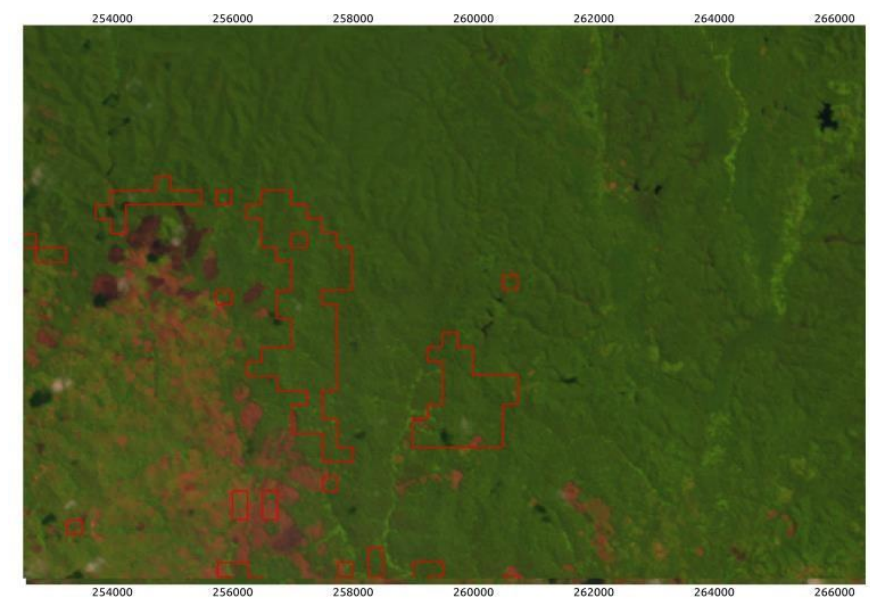


Figura 11: Esta es la misma imagen del año 2010 en donde se muestra que las áreas dentro de los polígonos de color rojo no están deforestadas.

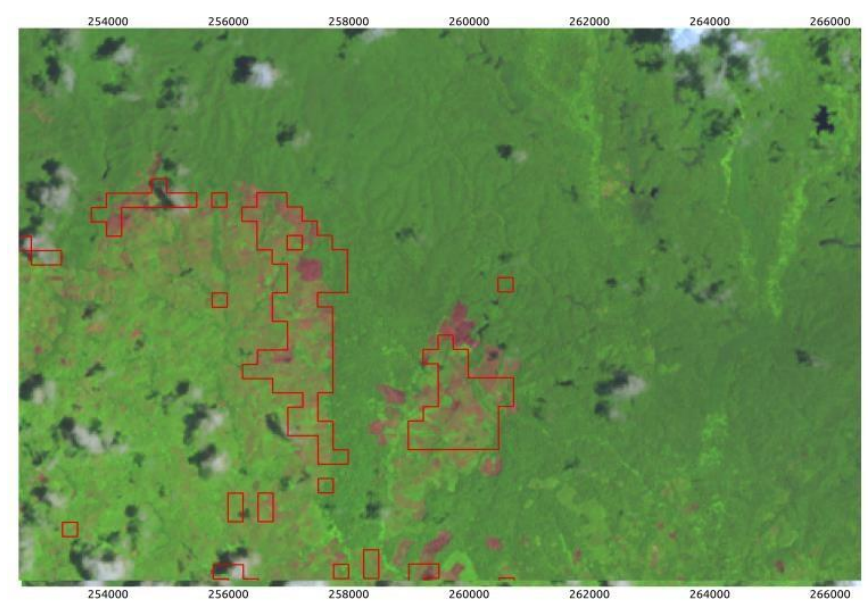

Figura 12: Al año 2013 se puede apreciar que hubo una deforestación en el año 2012 y que fue detectado por el método Los Promedios Anuales.

Veamos áreas deforestadas en el año 2013 para la misma zona de estudio.

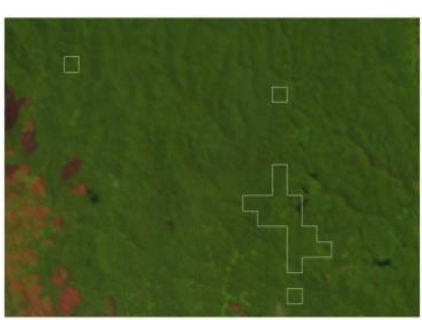

a).

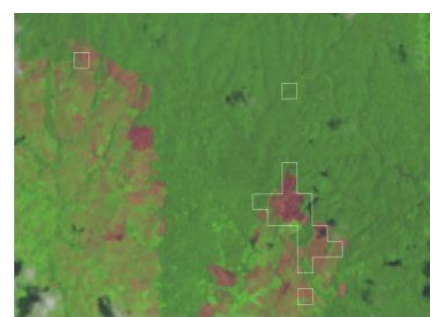

b).
Figura 13: Apreciamos que en la figura a) del año 2010 no hay deforestación, pero al año 2013 en la figura b) hubo cambio en el uso del bosque.

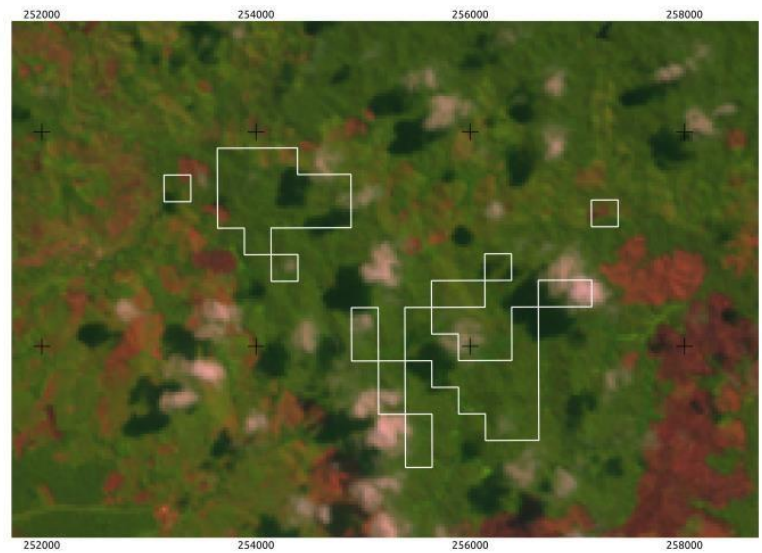

a).

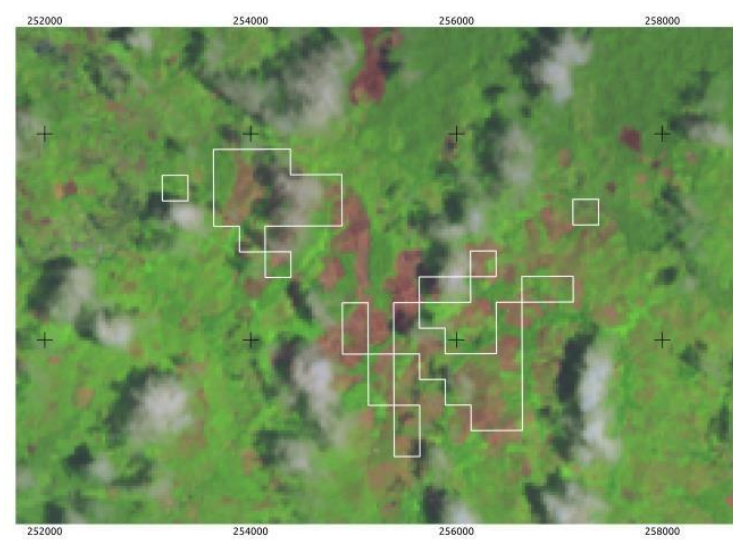

b).

Figura 14: Nuevamente vemos que al 2010 no hay cambio alguno dentro de los polígonos de color blanco (figura a), pero en el año 2013 los cambios son notorios figura $b$.

\section{Discusión de los resultados}

La metodología de Los Promedios Anuales mostró buenos resultados al detectar y cuantificar el cambio de uso de cobertura, lo que indica que es una muy buena opción si queremos monitorear a mediano y largo plazo los cambios en los bosques por perturbaciones antrópico o natural.

Es importante mencionar que, como cualquier método, tiene un margen de error y es que por la complejidad de los miles de series de tiempo, algunas de estas son detectadas como cambio, pero que en realidad si uno plotea la serie de tiempo y analizamos su tendencia, veremos que no hay ningún cambio en ella, por lo que solo nos queda decir que fueron detectadas debido a la complejidad de la misma. Por ello como solución a este problema, dentro de la metodología de los promedios Anuales, se incluyó un algoritmo que minimiza este problema y va eliminando aquellas series de tiempo que han sido detectadas como deforestación, pero que en su tendencia no hay ningún cambio brusco. 

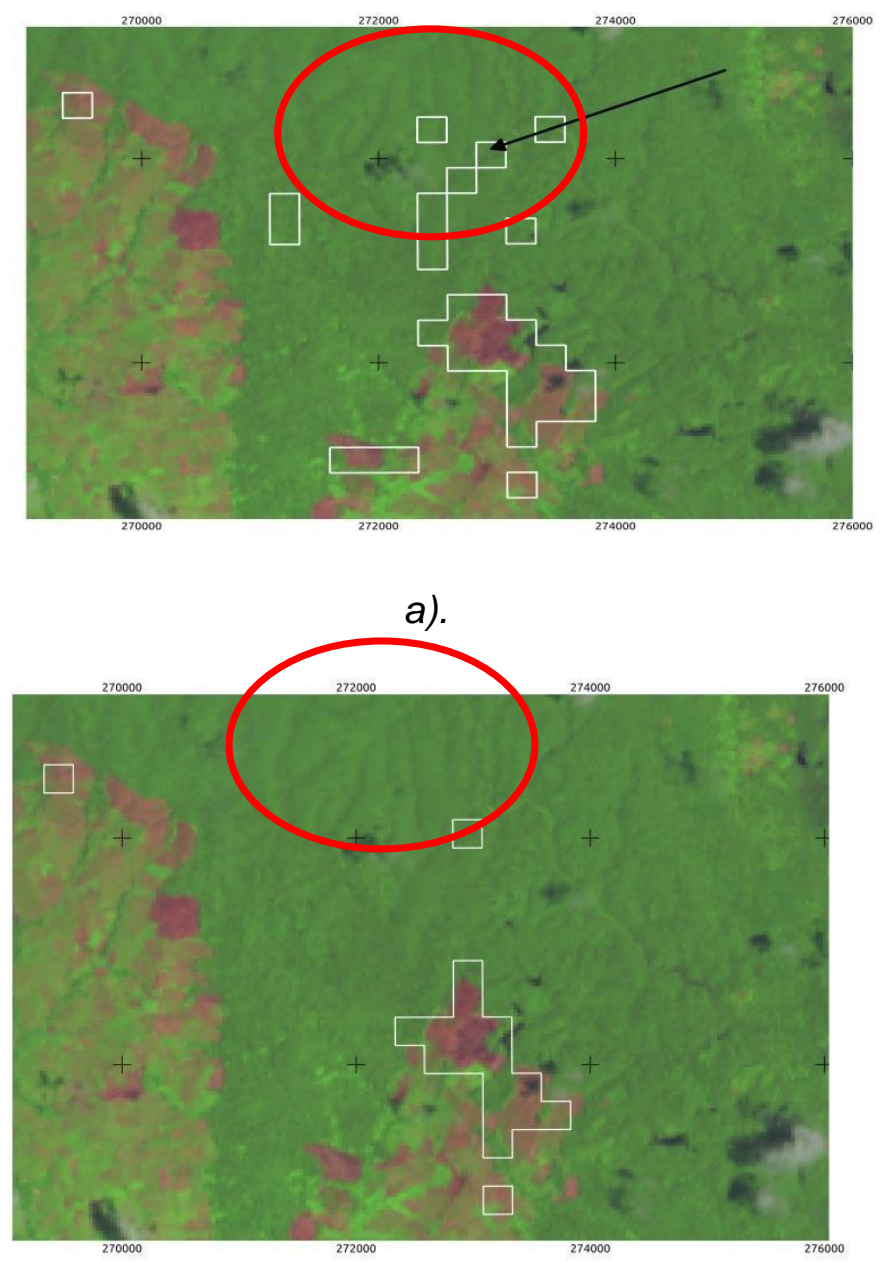

b).

Figura 15: Estos errores (figura a) debido a la complejidad de la serie de tiempo se minimizan (figura b) de tal manera que los resultados sean los más óptimos posibles.

\section{Conclusiones}

Los Promedios Anuales (LPA), metodología para detectar grandes cambios mediante la estadística de gráficos de control, resultó ser muy buena de acuerdo a los resultados mostrados líneas arriba. Posiblemente es necesario comprobar esta metodología en diversos tipos de cobertura, ya que esta fue probada en bosques tropicales - Amazonía peruana, en donde los valores del verdor de la vegetación son muy altas.

No se necesita un umbral para detectar si hubo o no un cambio en el uso de la cobertura, ya que trabaja en base a series de tiempo y es una metodología multitemporal en el que utiliza más de dos imágenes.

Al ser una metodología que detecta cambios a mediano y largo plazo, es necesario contar con muchos años de información (mayor a 10), para tener la certeza que los resultados obtenidos sean satisfactorios.

\section{Agradecimientos}

Mis agradecimientos al Laboratorio de Teledetección - LABTEL, de la Facultad de Ciencias Físicas por brindarme el apoyo necesario para publicar esta pequeña investigación y contribuir con una metodología que nos permita monitorear los bosques deforestados para que las instituciones encargadas de velar por ellas tomen conciencia y cartas en el asunto.

\section{Referencias}

[1] Peter J. Brockwell-Richard A. Davis (2002). Introduction to Time Series and Forescasting, pp. 1-40,317-333 Second Edition. EE.UU. Editorial Springer.

[2] Se Christian Kleiber - Achim Zeileis. (2011). Applied Econometrics with R, pp. 160-180. Baltimore-EE.UU. Editorial Spring.

[3] Jean-Francois Mas (2011). Aplicaciones del sensor MODIS para el monitoreo del territorio. Primera edición. México. Secretaría de Medio Ambiente y Recursos Naturales (SEMARNAT), Instituto Nacional de Ecología (INE), Universidad Nacional Autónoma de México (UNAM), Centro de Investigaciones en Geografía Ambiental (CIGA).

$$
\begin{aligned}
& \text { Dr. Josué Álvarez Borrego. } \\
& \text { Control }
\end{aligned}
$$

Estadísticos de Procesos.

[5] Humberto Gutiérrez Pulido. Román de la Vara. Control Estadístico para Calidad y Seis Sigma. 